Vol. 47 (1993) [435-442]

\title{
MULTIPLIERS ON SPACES OF FUNCTIONS ON COMPACT GROUPS WITH $P$-SUMMABLE FOURIER TRANSFORMS
}

\author{
Sanjiv Kumar Gupta, Shobha Madan and U.B. Tewari
}

Let $G$ be a compact abelian group with dual group $\Gamma$. For $1 \leqslant p<\infty$, denote by $A_{p}(G)$ the space of integrable functions on $G$ whose Fourier transforms belong to $\ell_{p}(\Gamma)$. We investigate several problems related to multipliers from $A_{p}(G)$ to $A_{q}(G)$. In particular, we prove that $\left(A_{p}, A_{p}\right) \varsubsetneqq \bigcap_{z<q<p}\left(A_{q}, A_{q}\right)$. For the circle group, we characterise permutation invariant multipliers from $A_{p}$ to $A_{r}$ for $1 \leqslant$ $r \leqslant 2$.

\section{INTRODUCTION}

Let $G$ be a locally compact abelian group with dual $\Gamma$. For $1 \leqslant p<\infty$, the space $A_{p}(G)$ is defined as:

$$
A_{p}(G)=\left\{f \mid f \in L^{1}(G), \widehat{f} \in L^{p}(\Gamma)\right\}
$$

with the norm $\|f\|_{A_{p}}=\|f\|_{L^{1}}+\|\hat{f}\|_{L^{p}}$. Then $A_{p}(G)$ is a commutative semi-simple Banach algebra with maximal ideal space $\Gamma$.

A function $\phi$ on $\Gamma$ is said to be a multiplier from $A_{p}$ to $A_{r}$ if $\phi \widehat{f} \in \widehat{A}_{r}$ for every $f \in A_{p}$. The set of multipliers from $A_{p}$ to $A_{r}$ is denoted by $\left(A_{p}, A_{r}\right)$. It is well-known that a continuous linear operator $T: A_{p} \rightarrow A_{r}$ commutes with translations in $G$ if and only if there exists a function $\phi$ on $\Gamma$ such that $(T f)^{\wedge}=\phi \widehat{f} \forall f \in A_{p}$. We shall denote by $\|\phi\|$ the operator norm of $T$. For a discussion of $\left(A_{p}, A_{r}\right)$ multipliers, we refer to the paper by Bloom and Bloom [1].

If $G$ is non-compact, then $\left(A_{p}, A_{p}\right)=\widehat{M}(G)[4]$. For a compact group $G$, if $1 \leqslant p \leqslant 2$, then $\left(A_{p}, A_{p}\right)=\ell_{\infty}$, since in this case $\widehat{A}_{p}=\ell_{p}(\Gamma)$. Further if $r \geqslant p$, then it is easy to see that $\left(A_{p}, A_{p}\right)=\left(A_{p}, A_{r}\right)$. Thus the cases of interest are when $p>2$ and $1 \leqslant r<p$.

In [10] Tewari and Gupta proved that for $1 \leqslant r<p$ and $2<p<\infty$

(a) $\left(A_{p}, A_{p}\right) \cap C_{0}(\Gamma) \varsubsetneqq\left(A_{r}, A_{r}\right) \cap C_{0}(\Gamma)$,

(b) $\bigcup_{r<p}\left(A_{p}, A_{p}\right) \subsetneq\left(A_{r}, A_{r}\right)$.

Received 25 May 1992

Copyright Clearance Centre, Inc. Serial-fee code: 0004-9729/93 \$A2.00+0.00. 
The following was stated as an open problem in [8].

If $2<p<\infty$, is $\left(A_{p}, A_{p}\right) \varsubsetneqq \bigcap_{r<p}\left(A_{r}, A_{r}\right) ?$

In this paper, we provide an affirmative answer to this problem (Theorem 2.1).

In general, the multiplier spaces $\left(A_{p}, A_{r}\right)$ are not known for all values of $\boldsymbol{p}$ and $\boldsymbol{r}$, $p>2$. However it is easy to see that certain sequences spaces are contained in them (see [1]).

If $1 \leqslant r \leqslant 2, r<p$, then clearly $\ell_{(r p) /(p-r)} \subseteq\left(A_{p}, A_{r}\right)$. However, for $p>2$ it is not known whether this contaiment is proper. In [10] it was shown that

(i) if $s>(r p) /(p-r)$, then $\ell_{d} \nsubseteq\left(A_{p}, A_{r}\right), 1 \leqslant r \leqslant 2<p<\infty$,

(ii) if $s>(2 p) /(p-2)$, then $\ell, \nsubseteq\left(A_{p}, A_{r}\right), 2<r \leqslant p<\infty$.

Using the idea of the proof of Theorem 2.1, we improve this result in Theorem 2.3.

In the last section we study the space of permutation invariant multipliers $\Pi\left(A_{p}, A_{r}\right)$ for the circle group $T$. (A multiplier $\phi \in\left(A_{p}, A_{r}\right)$ is said to be permutation invariant if $\phi \circ \pi \in\left(A_{p}, A_{r}\right)$ for all permutations $\pi$ of $\left.\Gamma\right)$. We show that if $1 \leqslant r \leqslant 2<p<\infty$ then $\ell_{(r p) /(p-r)}$ is precisely the set of permutation invariant multipliers from $A_{p}$ to $A_{r}$.

We shall need some results on pointwise multipliers from $\ell_{p}(\Gamma)$ to $\ell_{r}(\Gamma)$, were $\Gamma$ is discrete. The space $M\left(\ell_{p}, \ell_{r}\right)$ of pointwise multipliers consists of functions $\phi$ on $\Gamma$ such that $\phi f \in \ell_{r} \forall f \in \ell_{p}$. Using the reverse Hölder's inequality it is easy to see that

(i) $M\left(\ell_{p}, \ell_{r}\right)=\ell_{\infty}$ if $p \leqslant r$,

(ii) $M\left(\ell_{p}, \ell_{r}\right)=\ell_{(p r) /(p-r)}$ if $p>r$.

(see [1]).

\section{Proper inclusion in $\left(A_{p}, A_{r}\right)$-Spaces}

ThEOREM 2.1. Let $G$ be a compact abelian group and $p>2$. Then

$$
\left(A_{p}, A_{p}\right) \varsubsetneqq \bigcap_{q<p}\left(A_{q}, A_{p}\right)
$$

The proof of Theorem 2.1 depends on an interesting lemma about sequences spaces, which may be of independent interest and is suggested by the equality

$$
M\left(\ell_{(r p) /(p-r)}, \ell_{r}\right)=\ell_{p}, \quad 1 \leqslant r<p<\infty .
$$

We also use this lemma to improve certain results about $\left(A_{p}, A_{r}\right)$ multipliers due to Tewari and Gupta [10].

LEMMA 2.2. Let $I$ be an infinite set. Let $1 \leqslant r<p<\infty$ and $\phi \in \ell_{p}(I)$ be such that $\phi \notin \ell_{q}(I)$ for every $q<p$. Then there exists $\psi \in \bigcap_{t>(r p) /(p-r)} \ell_{t}(I)$ such that $\phi \psi \notin \ell_{r}(I)$ 
Proof: Clearly, we may assume $|\phi| \leqslant 1$ on $I$. Fix a positive integer $s>p / r$. Let $q_{j}=p-1 / j$ and choose $m_{0} \in \mathbb{N}$ such that $q_{j}>r$ for $j \geqslant m_{0}$. Now define, $\alpha_{j}=\left(r q_{j}\right) /\left(q_{j}-r\right), j \geqslant m_{0}$. Then $q_{j}$ increases to $p$ and $\alpha_{j}$ decreases to $(r p) /(p-r)$. Let $\left(a_{n}\right)_{n=1}^{\infty}$ be the support of $\phi$. Let $n_{0}=0$ and choose $n_{1}>1$ such that

$$
\sum_{n=1}^{n_{1}}\left|\phi\left(a_{n}\right)\right|^{q_{t}}>1
$$

By induction, construct an increasing sequence $\left(n_{j}\right)_{j=1}^{\infty}$ of integers such that

$$
\sum_{n=n_{j-1}+1}^{n_{j}}\left|\phi\left(a_{n}\right)\right|^{q_{j}}>1, \quad \forall j \geqslant 1
$$

Define

$$
\psi(a)= \begin{cases}\left|\phi\left(a_{n}\right)\right|^{p / \alpha_{j}} & \text { if } a=a_{n}, \quad n_{j-1}<n \leqslant n_{j}, \quad \forall j \geqslant 1 \\ 0 & \text { on } I \backslash\left\{a_{n}\right\}_{n=1}^{\infty} .\end{cases}
$$

Then if $k \geqslant m_{0}$, we have

$$
\begin{aligned}
\sum_{n=n_{k}+1}^{\infty}\left|\psi\left(a_{n}\right)\right|^{\alpha_{k}} & =\sum_{j=k}^{\infty} \sum_{n=n_{j}+1}^{n_{j+1}}\left|\phi\left(a_{n}\right)\right|^{\alpha_{k} p / \alpha_{j+1}} \\
& \leqslant \sum_{n=n_{k}+1}^{\infty}\left|\phi\left(a_{n}\right)\right|^{p}<\infty
\end{aligned}
$$

since $\alpha_{k}$ is decreasing and $|\phi| \leqslant 1$.

Therefore

$$
\psi \in \bigcap_{k=m_{0}}^{\infty} \ell_{\alpha_{k}}(I)=\bigcap_{t>(r p) /(p-r)} \ell_{t}(I) .
$$

Next, to see that $\phi \psi \notin \ell_{r}(I)$, consider

$$
\begin{aligned}
\sum_{n=1}^{\infty}\left|\phi\left(a_{n}\right)\right|^{r}\left|\psi\left(a_{n}\right)\right|^{r} & =\sum_{j=1}^{\infty} \sum_{n=n_{j-1}+1}^{n_{j}}\left|\phi\left(a_{n}\right)\right|^{p r / \alpha_{j}}\left|\phi\left(a_{n}\right)\right|^{r} \\
& \geqslant \sum_{j=1}^{\infty} \sum_{n=n_{j-1}+1}^{n_{j}}\left|\phi\left(a_{n}\right)\right|^{q_{j}}=\infty
\end{aligned}
$$

since $s>p / r$, and so $r+p r / \alpha_{j}<q_{j}, \forall j \geqslant 1$.

This completes the proof of the lemma. 
Proof of Theorem 2.1: Since $\bigcup_{q<q} A_{q} \varsubsetneqq A_{p}$ [9], there exists $f \in A_{p}$ such that $\widehat{f} \notin \ell_{q}$ for every $q<p$. Let $\phi=\widehat{f}$. Then $\phi$ satisfies the conditions of Lemma 2.2 with $r=2$. Hence there exists $\psi \in \bigcap_{t>(2 p) /(p-2)} \ell_{t}$ such that $\phi \psi \notin \ell_{2}$. For $2<q<p$, we have $(2 q) /(q-2)>(2 p) /(p-2)$, so that $\bigcap_{t>(2 p) /(p-2)} \ell_{t}=\bigcap_{p>q>2} \ell_{(2 q) /(q-2)}$. Hence $\psi \in \bigcap_{p>q>2} \ell_{(2 q) /(q-2)}$.

Since $\phi \psi \notin \ell_{2}$, there exists a function $\varepsilon$ on $\Gamma$ whose range is contained in $\{ \pm 1\}$ such that $\varepsilon \phi \psi \notin\left(L^{1}\right)^{\wedge}\left[2\right.$, Theorem 1.1]. Then $\varepsilon \psi$ belongs to $\bigcap_{p>q>2} \ell_{(2 q) /(q-2)} \subseteq$ $\bigcap_{p>q>2}\left(A_{q}, A_{q}\right)$, and $\varepsilon \psi \notin\left(A_{p}, A_{q}\right)$. This completes the proof of the theorem.

We now use Lemma 2.2 to improve some results of [10] mentioned in the introduction.

TheORem 2.3. Let $G$ be an infinite compact abelian group. Then

(a) $\bigcap_{\text {s }>(r p) /(p-r)} \ell, \nsubseteq\left(A_{p}, A_{r}\right), 1 \leqslant r \leqslant 2<p<\infty$.

(b) $\bigcap_{\text {. }>(2 p) /(p-2)} \ell, \nsubseteq\left(A_{p}, A_{r}\right), 2<r \leqslant p<\infty$.

Proof: (a) Since $\bigcup_{q<q} A_{q} \varsubsetneqq A_{p}$, there exists $f \in A_{p}$ such that $\hat{f} \notin \ell_{q}$ for every $q<p$. Hence by Lemma 2.2 we get $\psi \in \bigcap_{,>(r p) /(p-r)} \ell_{q}$ such that $\psi \hat{f} \notin \ell_{r}$. Thus
$\psi \notin\left(A_{p}, A_{r}\right)$.

(b) Using (a) for $r=2$ we get $\phi \in \bigcap_{,>(2 p) /(p-2)} \ell$, such that $\phi \notin\left(A_{p}, A_{2}\right)$. Hence there exists $f \in A_{p}$ such that $\phi \widehat{f} \notin \ell_{2}$. Now there exists a function $\varepsilon$ defined on $\Gamma$ with range in $\{ \pm 1\}$ such that $\varepsilon \phi \widehat{f} \notin\left(L^{1}\right)^{\wedge}$. Hence $\varepsilon \phi \notin\left(A_{p}, A_{r}\right)$ and $\varepsilon \phi \in$ $\bigcap_{0>(2 p) /(p-2)} \ell_{s}$.

It was mentioned in the introduction that if $1 \leqslant r \leqslant 2<p<\infty$ then the proper containment of $\ell_{(r p) /(p-r)}$ in $\left(A_{p}, A_{r}\right)$ is not known. In Theorem 2.4 below, we give a sufficient condition on a multiplier $\phi \in\left(A_{p}(T), A_{r}(T)\right)$ so that $\phi \in \ell_{(r p) /(p-r)}(\mathbb{Z})$.

It is easy to see that for $r \leqslant 2, \phi \in\left(A_{p}, A_{r}\right)$ if and only if $|\phi| \in\left(A_{p}, A_{r}\right)$. Further $\phi \in\left(A_{p}, A_{r}\right)$ if and only if $\psi(\gamma)=\phi(\gamma)+\phi(-\gamma) \in\left(A_{p}, A_{r}\right)$. Therefore it is sufficient to characterise non-negative, even multipliers from $A_{p}$ to $A_{r}$.

THEOREM 2.4. Let $1 \leqslant r \leqslant 2<p<\infty$ and let $\phi$ be a non-negative, even sequence on $\mathbb{Z}$ such that $\phi(n+1) \leqslant \phi(n), n>0$. Then $\phi \in\left(A_{p}(T), A_{r}(T)\right)$ if and only if $\phi \in \ell_{(p r) /(p-r)}(\mathbb{Z})$.

Proof: If $\phi \in \ell_{(r p) /(p-r)}$, clearly $\phi \in\left(A_{p}, A_{r}\right)$. For the converse, let $\phi \in$ 
$\left(A_{p}(T), A_{r}(T)\right)$. We may assume that $\phi(0)=0$. Let

$$
\psi_{m}(n)= \begin{cases}(\phi(n))^{r /(p-r)} & \text { on }[-m, m] \\ 0 & \text { otherwise. }\end{cases}
$$

Then

$$
\left\|\phi \psi_{m}\right\|_{\ell_{r}} \leqslant\|\phi\|\left(\left\|\bar{\psi}_{m}\right\|_{L^{1}}+\left\|\psi_{m}\right\|_{L_{p}}\right)
$$

Now by $[3,7.3 .3]$

$$
\begin{aligned}
\left\|\dot{\psi}_{m}\right\|_{L^{1}} & \leqslant C \sum_{n=1}^{m} \frac{\psi_{m}(n)}{n} \\
& \leqslant C \sum_{n=1}^{m}\left(\frac{1}{n^{p^{\prime}}}\right)^{1 / p^{\prime}}\left(\sum_{n=1}^{m}(\phi(n))^{p r /(p-r)}\right)^{1 / p} .
\end{aligned}
$$

Also,

$$
\begin{aligned}
\left\|\phi \psi_{m}\right\|_{l_{r}} & =\left(2 \sum_{n=1}^{m}(\phi(n))^{r}(\phi(n))^{r^{2} /(p-r)}\right)^{1 / r} \\
& =2^{1 / r}\left(\sum_{n=1}^{m}(\phi(n))^{p r /(p-r)}\right)^{1 / r}
\end{aligned}
$$

Hence, combining (2.5)-(2.7) we get

$$
\begin{aligned}
2^{1 / r}\left(\sum_{n=1}^{m}(\phi(n))^{p r /(p-r)}\right)^{1 / r} \leqslant\|\phi\| & \left\{C^{\prime}\left(\sum_{n=1}^{m}(\phi(n))^{p r /(p-r)}\right)^{1 / p}\right. \\
+ & \left.\left(\sum_{n=1}^{m}(\phi(n))^{p r /(p-r)}\right)^{1 / p}\right\} .
\end{aligned}
$$

It follows that

$$
\left(\sum_{n=1}^{m}(\phi(n))^{p r /(p-r)}\right)^{1 / r-1 / p} \leqslant\|\phi\|\left(1+C^{\prime}\right) 2^{-1 / r}
$$

where the constant $C^{\prime}$ is independent of $m$. Hence $\phi \in \ell_{p r /(p-r)}(\mathbb{Z})$. This completes the proof of the theorem. 


\section{Permutation invariant multipliers from $A_{p}$ to $A_{r}$}

In this section we study permutation invariant multipliers from $A_{p}$ to $A_{r}$ on the circle group. In general, as we have already seen, if $p \leqslant 2$, then $\left(A_{p}, A_{r}\right)=\ell_{r p /(p-r)}$ if $r<p$ and $\left(A_{p}, A_{r}\right)=\ell_{\infty}$ if $r \geqslant p$. Therefore we assume that $p>2$. The following theorem completely characterises $\Pi\left(A_{p}(T), A_{r}(T)\right), 1 \leqslant r \leqslant 2<p<\infty$.

Theorem 3.1. Let $1 \leqslant r \leqslant 2<p<\infty$, then

$$
\ell_{p r /(p-r)}(\mathbb{Z})=\Pi\left(A_{p}(T), A_{r}(T)\right)
$$

The proof of the above theorem depends on the following lemma:

LemmA 3.2. Let $2<p<\infty$ and $(a(n)) \in \ell_{p}(\mathbb{Z})$ be such that $a(n) \geqslant 0$ and $a(n)=a(-n) \forall n \in \mathbb{N}$. Then there exists a permutation $\pi$ of $\mathbb{Z}$ such that $a \circ \pi(n) \in$ $\left(L^{1}(T)\right)^{\wedge}$.

PROOF: Let $\pi$ be a permutation of $\mathbb{N}$ such that $a \circ \pi$ is decreasing on $\mathbb{N}$. Extend $\pi$ to $\mathbb{Z}$ by defining $\pi(-n)=-\pi(n) \forall n \in \mathbb{N}$ and $\pi(0)=0$. We show that $a \circ \pi \in\left(L^{1}(T)\right)^{\wedge}$. Clearly, $a \circ \pi(n) \geqslant 0, a \circ \pi(n)=a \circ \pi(-n) \forall n \in \mathbb{N}$ and $a \circ \pi(n)$ decreases to zero on $\mathbb{N}$. Also,

$$
\sum_{n=1}^{\infty} \frac{a \circ \pi(n)}{n} \leqslant\left(\sum_{n=1}^{\infty}(a \circ \pi(n))^{p}\right)^{1 / p}\left(\sum_{n=1}^{\infty} 1 / n^{p^{\prime}}\right)^{1 / p^{\prime}}<\infty
$$

Therefore by $[3,7.3 .3] a \circ \pi \in\left(L^{1}(T)\right)^{\wedge}$.

This completes the proof of the lemma.

ProOf of TheOREM 3.1: It is clear that $\ell_{p r /(p-r)}(\mathbb{Z}) \subseteq \Pi\left(A_{p}(T), A_{r}(T)\right)$. Conversely, suppose $(a(n)) \notin \ell_{p r /(p-r)}(\mathbb{Z})=M\left(\ell_{p}(\mathbb{Z}), \ell_{r}(\mathbb{Z})\right)$, then there exists a sequence $(b(n)) \in \ell_{p}(\mathbb{Z})$ such that $(a(n) b(n))$ does not belong to $\ell_{r}(\mathbb{Z})$.

Define

$$
c(n)=\max \left(\frac{1}{|n|}+|b(n)|, \frac{1}{|n|}+|b(-n)|\right) .
$$

Then $(c(n)) \in \ell_{p}(\mathbb{Z})$, and $(a(n) c(n)) \notin \ell_{r}(\mathbb{Z})$. Also $(c(n))$ satisfies the conditions of Lemma 3.2, hence there exists a permutation $\pi$ of $\mathbb{Z}$ such that $(c \circ \pi(n)) \in$ $\left(L^{1}(T)\right)^{\wedge}$. Therefore $(c \circ \pi(n)) \in \widehat{A}_{p}(T)$. It follows that $a \circ \pi \notin\left(A_{p}(T), A_{r}(T)\right)$ as $(a \circ \pi(n) c \circ \pi(n)) \notin \ell_{r}(\mathbb{Z})$.

This completes the proof of the theorem.

In the case $2<r \leqslant p<\infty$ we are not able to characterise $\Pi\left(A_{p}(T), A_{r}(T)\right)$. Observe that if $p>2$, then $\ell_{2 p /(p-2)} \subsetneq \Pi\left(A_{p}, A_{p}\right)$ since the constant function 1 on $\Gamma$ belongs to $\Pi\left(A_{p}, A_{p}\right)$. We prove the following theorem, characterising a subclass of $\Pi\left(A_{p}(T), A_{r}(T)\right)$. 
THEOREM 3.3. Let $2<r \leqslant p<\infty$. If $(a(n))$ is a sequence on $\mathbb{Z}$ such that $(a(n) \varepsilon(n)) \in \mathbb{\Pi}\left(A_{p}(T), A_{r}(T)\right)$ for every sequence $(\varepsilon(n))_{n \in Z}, \varepsilon(n)= \pm 1$, then $(a(n)) \in$ $\ell_{2 p /(p-2)}(\mathbb{Z})$.

PROOF: If on the contrary $(a(n)) \notin \ell_{2 p /(p-2)}(\mathbb{Z})=M\left(\ell_{p}(\mathbb{Z}), \ell_{2}(\mathbb{Z})\right)$, then there exists a sequence $(d(n)) \in \ell_{p}(\mathbb{Z})$ such that $d(n) \geqslant 0, d(n)=d(-n), d(n) \neq 0 \forall n \in \mathbb{N}$ and $(a(n) d(n)) \notin \ell_{2}(\mathbb{N})$. By Lemma 3.2, there exists a permutation $\pi$ of $\mathbb{Z}$ such that $(d \circ \pi(n)) \in \widehat{A}_{p}(T)$. Since $(a \circ \pi(n) d \circ \pi(n)) \notin \ell_{2}(\mathbb{Z})$, therefore there exists a sequence $(\varepsilon(n))_{n \in Z}, \varepsilon(n)= \pm 1$, such that $(\varepsilon(n) a \circ \pi(n) d \circ \pi(n)) \notin\left(L^{1}(T)\right)^{\wedge}$. Hence $(a(n) \varepsilon(n)) \notin \Pi\left(A_{p}(T), A_{r}(T)\right)$, a contradiction.

This completes the proof of the theorem.

REMARK 3.4. Lemma 3.2 can be viewed as an intermediate result between the following:

(i) (Helgason [5]): Let $G$ be a compact abelian group and $\phi$ a function on $\Gamma$. Then $\phi \in \ell_{2}$ if and only if $\phi \circ \pi \in \widehat{L}^{1}$ for every permutation $\pi$ of $\Gamma$.

(ii) (Kahane [7]): There exists a sequence $\phi \in C_{0}(\mathbb{Z})$ such that $\phi \circ \pi \notin\left(L^{1}(T)\right)^{\wedge}$ for any permutation $\pi$ of $\mathbb{Z}$.

The proof of Helgason's result gives the following result about $\left(A_{p}, A_{p}\right)$-multipliers:

THEOREM 3.5. (a) Let $p>2$ and $\phi \in \Pi\left(A_{p}, A_{p}\right) \cap C_{0}$. Then there exists a permutation $\pi$ of $\Gamma$ such that $\phi \circ \pi \in\left(A_{p}, A_{2}\right)$.

(b) $\Pi\left(L^{1}, L^{1}\right) \cap C_{0}=\ell_{2}$.

Proof: (a) Let $E=\{\gamma \in \Gamma \mid \phi(\gamma) \neq 0\}$. Since $\phi \in C_{0}, E$ is countable. If $E$ is finite then $\phi \circ \pi \in\left(A_{p}, A_{2}\right)$ for every permutation $\pi$ of $\Gamma$. So we assume that $E$ is infinite. Let $E_{1}$ be an infinite subset of $E$ such that

$$
\sum_{\gamma \in E_{1}}|\phi(\gamma)|^{2 p /(p-2)}<\infty
$$

Let $E_{2}=E \backslash E_{1}$. If $E_{2}$ is finite then for every permutation $\pi$ of $\Gamma \phi \circ \pi \in \ell_{2 p /(p-2)} \subseteq$ $\left(A_{p}, A_{2}\right)$. So we assume that $E_{2}$ is infinite. Choose a countably infinite $\Lambda_{2}$ subset $F_{1}$ of $\Gamma$ [6] and define $F_{2}=\Gamma \backslash F_{1}$. Let $\pi$ be a permutation of $\Gamma$ mapping $F_{1}$ onto $E_{2}$. We claim that $\phi \circ \pi \in\left(A_{p}, A_{2}\right)$. Let $f \in A_{p}$. Since $\phi \circ \pi \in\left(A_{p}, A_{p}\right), \widehat{g}=(\phi \circ \pi) \widehat{f} \in \widehat{A}_{p}$. Using Hölder's inequality and (3.7), we get

$$
\sum_{\gamma \in F_{2}}|\phi \circ \pi(\gamma)|^{2}|\widehat{f}(\gamma)|^{2}<\infty,
$$

since for $\gamma \in F_{2} \phi(\pi(\gamma)) \neq 0$ only if $\pi(\gamma) \in E_{1}$. Hence there exists an $h \in L^{2}$ such that

$$
\widehat{h}(\gamma)= \begin{cases}\phi \circ \pi(\gamma) \widehat{f}(\gamma), & \gamma \in F_{2} \\ 0, & \text { otherwise }\end{cases}
$$


Now $g-h \in L_{F_{1}}^{1}$. Since $F_{1}$ is a $\Lambda_{2}$ set, we have $g-h \in L^{2}$ [6]. Therefore, $g \in L^{2}$.

(b) In this case we choose the set $E_{1} \subset E$ such that

$$
\sum_{\gamma \in E_{1}}|\phi(\gamma)|^{2}<\infty
$$

Then proceeding as above we see that $\phi \circ \pi \in\left(L^{1}, L^{2}\right)=\ell_{2}[6]$. Hence $\phi \in \ell_{2}$.

\section{REFERENCES}

[1] L.M. Bloom and W.R. Bloom, 'Multipliers on spaces of functions with p-summable Fourier transforms', in Lecture Notes in Mathematics 1359 (Springer-Verlag, Berlin, Heidelberg, New York, 1987), pp. 100-112.

[2] R.E. Edwards, 'Changing signs of Fourier coefficients', Pacific J. Math. 15 (1965), 463-475.

[3] R.E. Edwards, Fourier Series: A modern introduction I (Holt, Rinehart and Winston, 1979).

[4] A. Figa-Talamanca and G.I. Gaudry, 'Multipliers and sets of uniqueness of $L^{p}$ ', Michigan Math. J. 17 (1970), 179-191.

[5] S. Helgason, 'Lacunary Fourier series on noncommutative groups', Proc. Amer. Math. Soc. 9 (1958), 782-790.

[6] E. Hewitt and K.A. Ross, Abstract harmonic analysis, Grundlehren der Math. Wiss., Band 152, Vol. II (Springer-Verlag, Berlin, Heidelberg, New York, 1970).

[7] J.P. Kahane, 'Sur les rearrangements des suites de coefficients de Fourier-Lebesgue', C.R. Acad. Sci. Paris 265A (1967), 310-312.

[8] U.B. Tewari, 'The multiplier problem', The Mathematics Student 51 (1983), 206-214.

[9] U.B. Tewari and A.K. Gupta, 'Algebras of functions with Fourier transform in a given function space', Bull. Austral. Math. Soc. 9 (1973), 73-82.

[10] U.B. Tewari and A.K. Gupta, 'Multipliers between some function spaces on groups', $B$ ull. Austral. Math. Soc. 18 (1978), 1-11.

Department of Mathematics Indian Inst. of Technology, Kanput

Kanpur 2080

India 